\title{
Analíticas de aprendizaje para mejorar la enseñanza y el seguimiento a través de entornos virtuales
}

\author{
Gabriela Sabulsky ${ }^{1}$ (D) @ \\ ${ }^{1}$ Universidad Nacional de Córdoba (UNC), Argentina
}

\begin{abstract}
Resumen. El presente artículo de revisión bibliográfica sobre el tema analíticas de aprendizaje es una recopilación de las principales ideas que sostienen algunos de sus impulsores. Las analíticas de aprendizaje son dispositivos tecnológicos que se incorporan a entornos virtuales de interacción, con el fin de registrar las huellas digitales que dejan quienes participan en ellos, creando grandes bases de datos. Nos proponemos identificar autores referentes en el campo, explicar qué son las analíticas de aprendizaje, reconocer su aplicación en entornos educativos cerrados y abiertos (redes y aplicaciones web) y concluir con una reflexión que pretende imaginar las analíticas al servicio del aprendizaje.
\end{abstract}

Palabras clave: Analítica de aprendizaje; sistemas de gestión del aprendizaje; redes sociales; metacognición.

\begin{abstract}
Analíticas de Aprendizagem para melhorar o ensino e o monitoramento por meio de ambientes virtuais

Resumo. O presente artigo de revisão bibliográfica sobre o tema Analíticas de Aprendizagem é uma compilação das principais ideias que sustentam alguns de seus impulsores. As Analíticas de Aprendizagem são dispositivos tecnológicos que são incorporados a ambientes virtuais de interação, a fim de registrar as marcas digitais deixadas por aqueles que participam do processo, criando grandes bancos de dados. A nossa proposta é identificar os principais autores no campo, explicar quais são as Analíticas de Aprendizagem, reconhecer sua aplicação em ambientes educacionais fechados e abertos (redes e aplicativos web) e concluir com uma reflexão que visa imaginar as Analíticas a serviço da aprendizagem.
\end{abstract}

Palavras-chave: Analítica da Aprendizagem; sistemas de gestão de aprendizagem; redes sociais; metacognição.

Learning Analytics to improve teaching and monitoring through virtual environments The present text is the result of a bibliographical review on the topic Analytics of Learning. Learning Analytics are technological devices that are incorporated into virtual environments of interaction, in order to register the fingerprints that leave participating in them, creating large databases. We aim to identify relevant authors in the field, to explain what the Learning Analyzes are, to recognize their application in closed and open educational environments (networks and web applications) and to conclude with a reflection that pretends to imagine the Analytics in the service of learning.

Keywords: Learning analytics; learning management systems; social networks; metacognition.

\section{Presentación}

El presente artículo es fruto de una revisión bibliográfica sobre el tema analíticas de aprendizaje, que pretende sistematizar algunos aportes teóricos y visualizar su potencialidad para la mejora de los procesos educativos. Las analíticas de aprendizaje son dispositivos tecnológicos ${ }^{1}$ que se incorporan a

${ }^{1}$ Del latín disposiuus ("dispuesto", un dispositivo es un aparato o mecanismo que desarrolla determinadas acciones. Su nombre está vinculado a que dicho artificio está dispuesto para cumplir con su objetivo. 
las plataformas educativas o redes sociales con el fin de registrar la actividad de los estudiantes, creando grandes bases de datos. La Society for Learning Analytics Research lo define como un campo de estudio y lo describe como "la medición, recolección, análisis y presentación de datos sobre los alumnos y sus contextos, con el propósito de comprender y optimizar el aprendizaje y los entornos en los que se produce" (Long y Siemens, 2011, p. 34). Suthers y Verbert (2013), por su parte, lo definen como un campo que se sitúa a medio camino entre las ciencias del aprendizaje, la investigación educativa y el uso de técnicas computacionales para capturar y analizar datos.

Este tema ha sido poco desarrollado en nuestro contexto académico por desconocimiento de los dispositivos técnicos para su aplicación, por presuponer que se desprende de una concepción mercantilista de la educación y porque la mayor parte de la producción teórica se encuentra en idioma inglés. Haciendo un esfuerzo por despejar ideas, nos interesa, a priori, identificar autores clave sobre el tema; explicar qué son las analíticas de aprendizaje; reconocer su aplicación en entornos cerrados, como son por ejemplo las plataformas educativas; $y$, en entornos abiertos de aprendizaje, como son los medios sociales, por mencionar algunas de sus aplicaciones; para cerrar con una reflexión que pretende imaginar la analítica al servicio del aprendizaje.

La metodología seguida para el presente artículo asumió el formato de revisión bibliográfica, en tanto pretendía conformar los antecedentes de un marco teórico para desarrollar posteriormente un trabajo de indagación. Por tanto, fue necesario, en primera instancia, definir el problema que delimitó la búsqueda. En tal sentido, nos propusimos rastrear los orígenes del concepto, sus principales impulsores y los significados que ellos proponen. Como criterio de búsqueda se estableció el nombre completo en inglés Learning Analytics. La búsqueda inicial se realizó a través de Google Scholar. Sin embargo, de la información obtenida, se seleccionaron sólo artículos incluidos en el Journal of Learning Analytics, por entender que se trata de una publicación clave, que desde el año 2014 se ha convertido en un referente en el tema. En una segunda fase, la búsqueda se realizó a través de autores relevantes: Shum Buckingham, George Siemens, Francisco-José García-Peñalvo y Erik Duval; la definición de estos nombres se realizó consultando a una especialista en el tema, quien además validó la selección realizada. La pregunta principal (a modo de hipótesis) que orientó la búsqueda fue: ¿cómo se definen las analíticas de aprendizaje desde el pensamiento de quienes introducen el concepto en el mundo académico? Recuperar el sentido original, más allá de sus interpretaciones y usos posteriores, nos permitió acercarnos a la potencialidad de la idea. Nos interesó analizar una temática poco explorada y encontrarle un sentido 
propio a nuestro contexto y prácticas de enseñanza en entornos virtuales, pensando estas herramientas como una oportunidad para transparentar los procesos que genera la relación entre aprendizaje y tecnologías.

\section{Relaciones de fuerza entre sujetos y tecnologías}

El impacto de las transformaciones macrosociales encuentra respuestas singulares en la educación superior; por ejemplo, la adopción explosiva de las tecnologías (Levine, 2006). Desde una perspectiva optimista sobre la incorporación de la tecnología en la educación, la UNESCO (1998) define líneas de acción a corto, mediano y largo plazo considerando la inclusión de tecnologías como un aspecto clave para pensar en una mejora y transformación de la educación superior. Sólo un par de años después de la aparición formalizada del proyecto Sociedad de la Información, concebido entre 1991 y 1994 (Becerra, 2010), la UNESCO propuso la inclusión de las nuevas tecnologías en la educación superior en los siguientes términos:

Los rápidos progresos de las nuevas tecnologías de la información y la comunicación seguirán modificando la forma de elaboración, adquisición y transmisión de los conocimientos. También es importante señalar que las nuevas tecnologías brindan posibilidades de renovar el contenido de los cursos y los métodos pedagógicos, y de ampliar el acceso a la educación superior. No hay que olvidar, sin embargo, que la nueva tecnología de la información no hace que los docentes dejen de ser indispensables, sino que modifica su papel en relación con el proceso de aprendizaje, y que el diálogo permanente que transforma la información en conocimiento y comprensión pasa a ser fundamental... (UNESCO, 1998).

Sin embargo, sería un reduccionismo analizar las transformaciones socioculturales sólo a partir del surgimiento de los desarrollos tecnológicos, por el contrario es conveniente vincular la emergencia y la consiguiente expansión de las tecnologías a un cierto número de procesos socioculturales, que contribuyen al arraigo y la transformación de la sociedad (Miege, 2010):

Entre determinaciones técnicas y los procesos de arraigo social de las herramientas y los servicios asociados, se establecen relaciones de dependencia y determinación entrelazadas... (p.23).

Estamos analizando en este punto la relación entre tecnología y transformaciones sociales para desarmar la idea del impacto tecnológico, metáfora que según Lévy (2007) ha sido mal utilizada. ¿Vienen las técnicas de otro planeta? ¿Es la tecnología un actor autónomo, separado de la sociedad y la cultura, una entidad pasiva y percutida por un agente exterior?, se pregunta este autor. Nos interesa señalar sobre este aspecto la relación 
dialógica entre lo técnico, lo social y lo cultural. Si una tecnología cobra importancia en el seno de una sociedad es porque sus miembros se apropian de ella, a la vez que la transforman y se transforman en un juego bidireccional (Burbules y Callister, 2001). Las prácticas sociales habilitan ciertos usos de tecnologías, a la vez que las tecnologías modifican esas mismas prácticas culturales que le dieron vida.

Sin embargo, este movimiento bidireccional no es neutral, según Lévy (2007) "las técnicas son portadoras de proyectos, de esquemas imaginarios, de implicaciones sociales y culturales muy variadas. Su presencia y su uso en tal lugar y en tal época cristalizan en unas relaciones de fuerza cada vez diferentes entre seres humanos" (p. 7).

Nos interesa resaltar esta cuestión, considerando que en la base del diseño y creación de todo dispositivo tecnológico; por ejemplo, en nuestro caso las analíticas de aprendizaje, hay ideas, proyectos, que cristalizan una relación de fuerzas tanto de quienes los diseñan como de quienes los usan. Según Doueihi (2010) la tecnología se diseña con una intencionalidad, posee una agenda de acciones y los usuarios nos movemos dentro de esos límites, siendo pocos los manipuladores en términos que pueden traspasar la frontera y modificar la propia tecnología.

Aparece entonces una suerte de determinismo tecnológico, que nos hace dependientes de lo que la tecnología nos ofrece, sin tener competencias para intervenir. Esto sucede, en parte, por la disociación entre los productores y consumidores/usuarios de la tecnología y de la dificultad asociada a ello para entender el lenguaje de las ciencias de la computación o programación. Según Lévy (2007) la fluidez y la mutación constante del lenguaje digital, explicaría, en parte, la sensación de impacto, de exterioridad y de extrañeza que invade a las personas cuando entran en relación con un artefacto nuevo, el cual modifica sus prácticas sociales y profesionales. Se trata, según Lévy, de un devenir colectivo complejo que se cristaliza alrededor de objetos materiales, de programas informáticos y de dispositivos de comunicación.

A decir verdad, cada uno de nosotros se encuentra poco o mucho en este estado de desposesión. La aceleración es tan fuerte y tan general que incluso los que se consideran estar más 'a la última' están, en diversos grados, desbordados por el cambio, pues nadie puede participar activamente en la creación de transformaciones del conjunto de las especificidades técnicas, ni siquiera seguirlas de cerca. (Lévy, 2007, p.12) 
Según Tobeña (2011), en una sociedad globalizada, profundamente fragmentada y desigual, regida por lógicas mercantilistas, la tecnología asume cada vez más poder sobre la existencia humana. Siguiendo este planteo, Sadin (2013) nos Ileva a pensar un poco más allá:

esa "familiaridad carnal" (con la tecnología) viene acompañada por una distancia creciente, por una forma de invisibilidad del proceso en curso. Esto es muy emblemático en lo que atañe a los Data Centers que contribuyen a modelar las formas de nuestro mundo y escapan a toda visibilidad. Es una necesidad técnica. Sin embargo, esa tensión señala lo que se está jugando en nuestro medio ambiente digital contemporáneo: por un lado, una impregnación continua de los sistemas electrónicos, y, por el otro, una forma de opacidad sobre los mecanismos que la componen (párr. 15).

Por tanto, si esto es así, podemos imaginar que la relación de fuerzas entre las tecnologías y los sujetos es desigual, que existe un progresivo control de la tecnología sobre los actos de los usuarios; cuestión, que se irá incrementando con el paso del tiempo. Así lo dice el autor, llevándonos a plantear una delegación progresiva de los actos humanos a los sistemas inteligentes:

Esta dimensión en curso se amplificará en los próximos años. Además, nuestras vidas individuales y colectivas están cada vez más reorientadas por sistemas que nos conocen con mucha precisión, que nos sugieren ofertas hiper individualizadas, que nos aconsejan este u otro comportamiento. Por medio del uso de nuestros protocolos de interconexión se opera una cuantificación continua de los gestos, la cual autoriza un "asistente robotizado" expansivo de las existencias (Sadin, 2013, párr. 7).

Si esto es cierto, parafraseando a Sadin (2013), nos preguntamos entonces ¿qué margen tenemos para transformarnos en manipuladores con la tecnología?, ¿podemos acercarnos a las funcionalidades técnicas, revelar sus intenciones y hacer un uso inteligente de la técnica?

Los dispositivos tecnológicos son -desde esta perspectiva que estamos planteando en nuestro trabajo- coercitivos y productivos: limitan las clases de actividades que pueden llevarse a cabo, pero también instituyen nuevas prácticas. Nos interesa entonces-como punto de partida-en el próximo apartado, definir qué son las analíticas de aprendizaje para empezar a pensar en este dispositivo, el cual nos imaginamos como coercitivo y productivo en las prácticas educativas de los entornos virtuales. 


\section{Acerca de las analíticas de aprendizaje}

Las analíticas de aprendizaje son definidas por Suthers y Verbert (2013) como un campo de investigación, con su consecuente herramental técnico para la recolección de datos. En el que se pretende, como campo de indagación, recolectar y analizar datos sobre las acciones que realizan los estudiantes en un entorno virtual de aprendizaje, con el fin de mejorar y adaptar las propuestas educativas virtuales. El Informe Horizon del The New Media Consortium y la Universitat Oberta de Catalunya plantean su implantación de cuatro a cinco años, y define que:

Las analíticas de aprendizaje consisten en la interpretación de un amplio rango de datos producidos y recogidos acerca de los estudiantes para orientar su progresión académica, predecir actuaciones futuras e identificar elementos problemáticos. El objetivo de la recolección, registro, análisis y presentación de estos datos es posibilitar que los profesores puedan adaptar de manera rápida y eficaz las estrategias educativas al nivel de necesidad y capacidad de cada alumno. Aun, en sus primeras etapas de desarrollo, las analíticas de aprendizaje responden a la necesidad de llevar a cabo el seguimiento y control de la actividad en el campus para la toma de decisiones estratégicas. Por otro lado, pretenden aprovechar la gran cantidad de datos producidos por los estudiantes en actividades académicas (Durall et al., 2012, p.13)

Al parecer este pronóstico de implantación se ha cumplido en algunas partes del mundo, siendo los países europeos y anglosajones los que cuentan con mayor experiencia sobre esta temática. En nuestro contexto educativo latinoamericano no es un tema del todo instalado, siendo aún pocas las experiencias que aprovechan la integración de analíticas de aprendizaje como estrategia de seguimiento y mejora.

Tratando de entender qué son las analíticas de aprendizaje recurrimos al Journal of Learning Analytics ${ }^{2}$, identificando algunos artículos de referentes teóricos, entre ellos se destacan: Buckingham (2012); Buckingham y Deakin (2016); Buckingham y Ferguson (2012); Siemens (2013); Gómez-Aguilar, García-Peñalvo y Therón (2014); García-Holgado y García-Peñalvo (2017). Estos autores coinciden en señalar lo fértil del campo aún en pleno desarrollo y la necesidad de migrar hacia una mirada más cualitativa de la información/ datos recogidos. En especial, la revisión de Simon Buckingham Shum del Knowledge Media Institute, así como de Rebecca Ferguson del Institute of Educational Technology de la Open University y de George Siemens del Technology Enhanced Knowledge Research Institute, Universidad de Athabasca (los tres del ámbito anglosajón), nos permiten reconstruir históricamente el

${ }^{2}$ Disponible en http://epress.lib.uts.edu.au/journals/index.php/JLA/issue/archive 
campo y entender cuáles son sus preocupaciones actuales. También GarcíaPeñalvo de la Universidad de Salamanca, como director del grupo de investigación Grial (Grupo de investigación en Interacción y e-learning), se suma a la perspectiva de los tres primeros autores mencionados.

De hecho, según estos autores, un hito clave en la conformación de un campo de investigación y producción, fue la realización de la $1^{\text {a }}$ Conferencia Internacional sobre Aprendizaje Analytics \& Knowledge, llevada a cabo en 2011, la cual logró formalizar a la comunidad académica sobre el tema. En ella se define la analítica de aprendizaje como:

la medición, recopilación, análisis y reporte de datos sobre los alumnos y sus contextos, con el propósito de comprender y optimizar el aprendizaje y los entornos en los que se produce (Buckingham y Ferguson, 2012 p. 4).

O bien como:

uso de estos datos y de cualquier otra observación adicional de la que se pueda obtener información, para tener un impacto directo sobre los estudiantes, los profesores y el proceso de aprendizaje (Long y Siemens, 2011).

Es interesante recuperar el foco puesto en el estudiante como beneficiario directo de la implementación de las analíticas de aprendizaje, esto es algo coincidente en todas las definiciones que encontramos de los autores citados.

Las analíticas de aprendizaje surgen en el ámbito de las organizaciones empresariales con la intención de conocer el comportamiento del consumidor externo. Este origen empresarial es uno de los factores que ha generado mayor resistencia, siendo el ámbito educativo uno de los más rezagados en tomarlo para fines propios. Según Buckingham y Ferguson (2012) las analíticas de aprendizaje tiene sus raíces en dos esfuerzos informáticos claves:

- La inteligencia de negocios, centrada en herramientas computacionales para mejorar la toma de decisiones organizativas, mediante la fusión eficaz de datos recopilados a través de diversos sistemas. La primera mención del término «análisis del aprendizaje» que hemos encontrado se relaciona con la inteligencia de negocios sobre productos y servicios de e-learning (Mitchell y Costello, 2000).

- La minería de datos, también Ilamada Knowledge Discovery in Databases (KDD), es el campo que se ocupa de emplear grandes cantidades de datos para apoyar el descubrimiento de información nueva y potencialmente útil (Piatetsky-Shapiro, 
1995). Este campo reúne muchas corrientes de investigación en computación, incluyendo redes neuronales artificiales, aprendizaje bayesiano, construcción de árboles de decisión, aprendizaje basado en instancias, programación lógica, inducción de reglas y algoritmos estadísticos (Romero y Ventura, 2007, p. 3 y 4)

Otro antecedente importante mencionado por Buckingham y Ferguson (2012) es el proyecto Signals de la Universidad de Purdue realizado durante el año 2010:

un ejemplo estrella del campo de la aplicación exitosa de la analítica académica, reportando niveles significativamente más altos y tasas de retención que las observadas en los grupos control (Arnold, 2010; Pistilli y Arnold, 2012). El proyecto extrae datos de un VLE y combina esto con el modelado predictivo para proporcionar un semáforo rojo / ámbar / verde en tiempo real a los estudiantes y educadores, ayudando al personal a intervenir de manera oportuna donde sea más beneficioso, y dando a los estudiantes un sentido de su progreso (p. 4)

Siguiendo al mismo autor, nos muestra cómo desde la minería de datos se trata de vincular las analíticas de aprendizaje al ámbito educativo. Buckingham y Ferguson (2012) citan a Baker y Yacef (2009), al definir la Minería de Datos Educativos (EDM) como:

una disciplina emergente, relacionada con el desarrollo de métodos para explorar los tipos únicos de datos que provienen de los entornos educativos, y el uso de estos métodos para entender mejor a los estudiantes y los entornos en que aprenden. Las primeras publicaciones de EDM se pueden encontrar desde 1995, pero una visión general del campo muestra que los proyectos de investigación se multiplicaron después de la adopción generalizada de entornos virtuales de aprendizaje (VLEs) a principios del siglo XXI. (Romero y Ventura, 2007 citado en Buckingham, 2012).

Según Gómez-Aguilar et al. (2014) citando a Siemens (2011-2012) destacan la relación entre las comunidades de las analíticas de aprendizaje y EDM en tanto ambas comunidades:

creen firmemente en la realización de investigaciones y aplicaciones que beneficien a los estudiantes, así como la mejora de las ciencias del aprendizaje. El mismo Siemens (2013) aclara que "mientras la LA está más preocupada por la toma de conciencia y la acción, la minería de datos educativos (EDM) se centra más en el desarrollo de métodos para "explorar los tipos únicos de datos que provienen de entornos educativos (GómezAguilar et al., 2014, p. 238) 
En base a este avance, Buckingham y Ferguson (2012) dicen:

Evidentemente, esto encapsula las líneas de todos los campos anteriores, reflejando la convergencia interdisciplinaria del tema; pero, en contraste con la investigación más teórica o la experimentación artificial que podría publicarse en algunos de los campos anteriores, se hace hincapié en el impacto del aprendizaje auténtico en el contexto del mundo real, a través del uso de herramientas prácticas (Long y Siemens, 2011). (Citado por Buckingham, 2012, p. 5).

Siemens (2013), por su parte, señala que las analíticas de aprendizaje se han desarrollado lo suficiente, a través de conferencias, revistas, institutos de verano y laboratorios de investigación, para considerarlo como un campo de investigación emergente.

Para avanzar un poco más en la comprensión de qué son y qué aportan las analíticas, la clasificación de Buckingham y Ferguson (2012), propuesta en Learning Analytics, informe publicado por UNESCO, permite distinguir capas o niveles de análisis. El autor distingue tres niveles: micro (a nivel del aula), meso (a nivel institucional) también definidas como analíticas académicas (Gómez-Aguilar et al., 2014) y macro (cuando remiten a un Estado, región, provincia). El nivel micro posibilita según el autor identificar a los estudiantes en riesgo y posibilitar intervenciones oportunas y proporcionar a los estudiantes una visión de sus propios hábitos de aprendizaje y recomendaciones para mejorar.

Entendemos que el nivel micro permitiría reconstruir, a manera de una fotografía, las huellas digitales que dejan grabadas los estudiantes en los entornos virtuales de aprendizaje; por ejemplo, identificando con qué recursos interactúan más, su ingreso a los foros, si escriben en ellos o sólo leen, si consultan el calendario, si ven videos, si bajan los materiales de estudio, si ingresan frecuentemente al aula virtual o sólo de forma esporádica, etc.

A través del uso de dispositivos móviles, sistemas de gestión del aprendizaje (LMS) y medios sociales, una mayor parte del proceso de aprendizaje genera senderos digitales. Un estudiante que inicia sesión en un LMS deja miles de puntos de datos, incluyendo patrones de navegación, pausas, hábitos de lectura y hábitos de escritura. Estos datos pueden ser puntos ambiguos y requieren exploración adicional para entender lo que significa una prolongada pausa de lectura (quizás el estudiante esté distraído o comprometido en otras tareas, o tal vez el estudiante está luchando con un concepto desafiante en el texto), pero para los investigadores, las ciencias del aprendizaje y la educación en general, los senderos de datos ofrecen una oportunidad para explorar el aprendizaje desde nuevos y múltiples ángulos" (Siemens 2013, p. 2). 


\section{Las analíticas de aprendizaje integradas a los sistemas de gestión del aprendizaje y medios sociales}

Un sistema de gestión de aprendizaje (LMS) es un software que permite organizar una propuesta educativa a distancia, permitiendo la interacción sincrónica y asincrónica entre profesores y estudiantes, y estudiantes entre sí. Entre sus funciones encontramos: la gestión de usuarios, distribución de recursos, materiales y actividades de aprendizaje, el seguimiento del proceso de aprendizaje, la evaluación y el proponer espacios de comunicación como foros, chats, videoconferencias, generar informes, entre otros.

Algunos ejemplos de sistemas de gestión del aprendizaje (o entornos virtuales de aprendizaje) son Moodle, Carolain, Sakai o Blackboard. Estos dispositivos tecnológicos disponen de un conjunto de herramientas que permiten el registro de senderos digitales en términos de lo que plantea Siemens, es decir de registro de datos a nivel micro. Sin embargo, para ampliar la base de registro se suelen agregar componentes extras, entre ellos podemos mencionar algunos módulos:

- Google analytics es un sistema de análisis y estadísticas gratuito que sirve para analizar diferentes aspectos del funcionamiento de una página o sitio web.

- Smartklass, es un plug-in para plataformas de aprendizaje online como Moodle, Sakai o Blackboard, que convierte los complejos datos estadísticos, en completos y a la vez sencillos informes que hacen un análisis del proceso de aprendizaje que se está produciendo en el entorno virtual.

- GISMO es una herramienta de monitorización gráfica interactiva que proporciona al profesorado una visualización útil de las actividades de los estudiantes en los cursos en línea.

- Por último, es posible cruzar datos de base y generar información significativa junto a la que provee la propia plataforma Moodle a través de: https://moodle.org/plugins/local analytics

En esta línea, los dispositivos tecnológicos posibilitan registrar cada vez con más detalle las actividades realizadas por los estudiantes. De hecho, en la actualidad se acumulan grandes cantidades de datos, que apenas están explorados por los mismos estudiantes, por sus profesores o por los gestores pedagógicos. Es difícil encontrar experiencias educativas que tomen estos registros como parte del seguimiento de alumnos o que los apliquen a procesos de retroalimentación del estudiante. 
Ahora bien, hasta aquí estamos planteando recuperar la huella de un estudiante en términos solitarios, en su interacción con el LMS (como sinónimo de aulas virtuales), este entorno más bien cerrado es un espacio que reproduce en parte las características de las aulas físicas de cuatro paredes, se dispone en ellas de información y actividades para favorecer el aprendizaje. Sin embargo, se observa una tendencia creciente a vincular las aulas virtuales con entornos virtuales abiertos, como es el caso de redes sociales o aplicaciones colaborativas tipo google drive, por mencionar un ejemplo. Esta vinculación, entre dispositivos tecnológicos cerrados (aulas virtuales) y abiertos (redes y aplicaciones web), refuerzan la condición indiscutible del carácter social del aprendizaje y nos plantea la necesidad de reconocer las huellas digitales de los estudiantes en ambos contextos, justamente por entender que es en la relación entre ambos contextos donde se produce el aprendizaje.

Se abre un terreno aún más inexplorado, aquel que se vincula a la necesidad de reconstruir las huellas digitales de los senderos colaborativos. Revisamos a partir de ahora las analíticas de aprendizaje social que centran la atención en los elementos de aprendizaje, que entran a ser relevantes cuando se aprende en una cultura participativa en línea.

En particular, el enfoque de SLA se centra en los procesos en los que los alumnos no son solitarios y no necesariamente realizan trabajo para ser identificados, sino que participan en la actividad social, ya sea interactuando directamente con otros (por ejemplo) o utilizando plataformas en las que sus rastros de actividad serán experimentados por otros (por ejemplo, publicación, búsqueda, etiquetado o calificación) (Buckingham y Ferguson, 2012, p. 5)

¿Con quién se aprende en los entornos virtuales?, ¿cómo entender la interacción social en ciertas condiciones pedagógicas?, ¿cómo se da la experiencia distribuida, la colaboración de los estudiantes en ámbitos virtuales? Al parecer, las analíticas de aprendizaje social nos permiten registrar las huellas del proceso social y colectivo que supone el aprendizaje. Los sistemas de gestión del aprendizaje (LMS), Moodle por ejemplo, ofrecen entornos de trabajo colaborativo a en foros y wikis a partir de los cuales se pueden reconocer conexiones (fuertes o débiles) entre un estudiante y su grupo de compañeros, entre estudiantes y tutores, y entre las comunidades de aprendizaje y los recursos de aprendizaje (Jones y Steeples, 2003 citado por Buckingham y Ferguson, 2012). Además podemos mencionar SNAPP (Social Networks Advancing Pedagogical Practice) que es un programa basado en el aprendizaje universitario, que analiza las redes sociales formadas dentro de los sistemas de gestión de aprendizaje. 


\section{Según Buckingham y Ferguson (2012):}

Los múltiples usos del análisis de redes sociales aplicables al aprendizaje social, incluyen la detección de comunidades dentro de las redes (Clauset, Newman y Moore, 2004; Fortunato, 2010); identificación de tipos de subconjuntos dentro de una red donde existe un nivel de cohesión y depende de propiedades tales como proximidad, frecuencia y afinidad u otras propiedades (Reffay y Chanier, 2003); investigación de la densidad de las redes sociales (Borgatti, Mehra, Brass y Labianca, 2009); y la exploración de la centralidad de los individuos dentro de una red (Wasserman y Faust, 1994) (Buckingham y Ferguson, 2012).

Si tomamos la interacción de los alumnos en un LMS como parte de una comunidad, puesto que son estudiantes con objetivos e intereses comunes, que construyen vínculos de pertenencia con esa comunidad, sostenida a partir de una red de interacciones virtuales, es de nuestro interés acercarnos a explorar la centralidad de cada estudiante dentro de la red que lo contiene. Buckingham y Ferguson proponen diferentes niveles o escalas: o miramos desde la red o miramos desde el individuo; es decir, dos perspectivas: egocéntrica y toda la red.

Las redes egocéntricas se describen desde el punto de vista del individuo, que se sitúa en el centro de una serie de relaciones formalmente e informalmente conectadas con el aprendizaje.

Estudiar las redes de esta manera puede ayudar a identificar a las personas de las cuales un individuo aprende, donde pueden surgir conflictos de comprensión y qué factores contextuales influyen en el aprendizaje. Una visión de toda la red, por otro lado, considera la distribución de la información y el desarrollo del aprendizaje a través de un conjunto de personas. En este caso, el análisis puede caracterizar la red en términos de su carácter, intereses y prácticas. Esta visión de toda la red es capaz de tomar "los resultados de las conexiones de pares para describir lo que mantiene unida a la red (Haythornthwaite y De Laat, 2010, p.189) (citado por Buckingham y Ferguson 2012 p. 5).

Además del análisis de las redes que se van configurando, según los autores que usamos como referencia, se puede combinar el análisis de redes con el análisis de contenido, registrando no sólo quién está hablando con quién, sino de qué están hablando y por qué están hablando de esta manera (De Laat, Lally, Lipponen y Simons, 2006; Hirst, 2011). Buckingham y Ferguson (2012) mencionan dos líneas de indagación a través de analíticas de aprendizaje social, a la primera la definen como de "disposición de aprendizaje" y a una segunda como "análisis de contenido", cada una con sus dispositivos tecnológicas que permiten producir datos. Al revisar la primera línea de investigación, sobre disposiciones, encontramos una serie de herra- 
mientas aplicadas en investigaciones de carácter internacional (ELLIment), que intentan dar cuenta de disposiciones contextualizadas, algunas de ellas son: curiosidad crítica, creación de significado, dependencia y fragilidad, creatividad, relaciones / interdependencia y conciencia estratégica (Buckingham y Deakin, 2012). Su carácter personal y situado limita a poder tomar estas disposiciones como generales.

Por otro lado, el análisis de contenidos desde una perspectiva más técnica, pretende examinar, indexar y filtrar activos multimedia en línea, con la intención de guiar a los estudiantes a través del océano de los recursos potenciales disponibles para ellos. Esta línea tiene actualmente un gran desarrollo. Nos interesa revisar con más detenimiento una línea de trabajo que intenta vincular el análisis de contenido con elementos más cualitativos, que permitan reconocer las formas de apropiación del contenido en los contextos sociales de aprendizaje, por ejemplo un foro. Hay antecedentes en esta dirección según Buckingham y Ferguson (2012).

\section{Imaginar las analíticas al servicio del aprendizaje}

Coll, Engel y Niño (2017) explican que mientras algunos estudios han puesto el foco en el diseño de algoritmos, que automatizan la minería de datos (Romero et al., 2007) y en la elaboración de propuestas visuales para presentar los resultados de los análisis (Mazza y Dimitrova, 2007; De la Fuente-Valentín y Burgos, 2015); otros estudios se han dirigido a analizar, cómo una analítica del aprendizaje puede proporcionar a los profesores una visión profunda del proceso de aprendizaje (Fidalgo-Blanco et al., 2015; Rodríguez-Triana et al., 2015). Centrados en esta última perspectiva, Kruse y Pongsajapan (2016) consideran que las analíticas de aprendizaje tienen actualmente dos grandes dificultades:

Hay, sin embargo, dos problemas potenciales con el matrimonio de los LMS y la analítica de aprendizaje. Primero es la suposición que tales sistemas se utilizan como sitios de aprendizaje significativo. Muchos LMS son ampliamente utilizados por los estudiantes, pero la profundidad de la interacción puede ser bastante superficial-limitada a la verificación de los resultados del examen o descargar el material para el curso. Además, hay una suposición de que los LMS son espacios para el aprendizaje autónomo. El enfoque para el aprendizaje analítico en LMS generalmente ignora la actividad fuera de estos sistemas, con el resultado de que sólo una pequeña porción del aprendizaje del estudiante y el compromiso se está captando. (p. 3) 
Dando por cierta esta afirmación, vale la pena reconocer que el escenario virtual de aprendizaje no podrá nuevamente encerrarse en un aula, aunque ahora sea virtual. En tal sentido, las analíticas de aprendizaje tienen el desafío de recoger datos que muestren la articulación entre los espacios cerrados (aula virtual) y abiertos (medios sociales), entendiendo que el aprendizaje se produce a partir del complemento de ambos entornos.

En segundo lugar, tales sistemas tienden a privilegiar al administrador más que al estudiante -o incluso al docente. El enfoque de la analítica de aprendizaje parece fijarse en una escala institucional más que en una escala humana. Queda por ver si los estudiantes y profesores serán vistos como participantes plenos en los mecanismos de análisis de aprendizaje de estos sistemas o como generadores involuntarios de datos para los administradores y las compañías detrás de estos LMS. (p. 4)

Esta preocupación lleva a estos autores a proponer la analítica al servicio del aprendizaje, al transformarla en una práctica caracterizada por un espíritu de cuestionamiento e indagación, lo que supone al estudiante como co-intérprete de sus propios datos y, tal vez, incluso como participante en la identificación y recolección de esos datos. "En este escenario, el estudiante toma conciencia de sus propias acciones en el sistema y utiliza esos datos para reflexionar y potencialmente cambiar su comportamiento" (Kruse 26 y Pongsajapan, 2016, p. 4). Un ejemplo es la investigación de Coll et al. (2017) quienes diseñan una analítica de aprendizaje basada en el enfoque de la Influencia Educativa Distribuida, Ios resultados indican que, en general, la información proporcionada a los participantes sobre su actividad tiene un impacto en el proceso colaborativo. Este ejemplo, de reciente publicación, abre un camino de indagación, dado que muestra cómo la analítica puede ponerse al servicio del aprendizaje.

Hemos mostrado a través de nuestro recorrido un estado de situación donde se cruzan preocupaciones sobre la mejora (personalización) de los procesos de aprendizaje con dispositivos tecnológicos, que permiten su registro y análisis. La investigación de Coll et al. (2017) señalada en el último ejemplo, considera que las analíticas de aprendizaje se pueden construir sobre la base de teorías, que los indicadores que se utilicen para evaluar la actividad se definen por quienes participan en la indagación, y que la información obtenida puede ser un insumo para los mismos estudiantes, quienes además pueden participar en todo el proceso de recogida y análisis de datos. 
Por tanto, entendemos que este es un camino a profundizar. En principio será necesario diseñar propuestas educativas que den cabida a los procesos de metacognición como estrategias de enseñanza, luego adecuar los dispositivos tecnológicos a esas necesidades educativas, y posteriormente será preciso habilitar al estudiante con los datos que el mismo sistema produce.

De esta manera el peso de la tecnología sobre las propuestas educativas, quedaría sujeta a la intervención crítica de quienes la usamos y consumimos, para transformarnos en productores inteligentes a través de ella.

\section{Conclusiones}

A través de la revisión bibliográfica se logró identificar algunas contribuciones claves para conocer el aporte que las analíticas de aprendizaje pueden realizar al campo educativo, advirtiendo acerca de la no neutralidad de la técnica.

Al describir los niveles de análisis de las analíticas, se sugiere el nivel micro para identificar las actividades dentro de las aulas virtuales y también la necesidad de incluir el registro de los espacios abiertos; es decir, las acciones que los mismos estudiantes realizan en sus redes sociales. En ese sentido, la potencialidad de las analíticas parece indiscutible: los dispositivos técnicos producen información más que necesaria a la hora de diseñar y evaluar las propuestas educativas. Ahora bien, como se plantea en el último apartado, esos datos deben ser analizados en base a teorías que ayuden a comprender los complejos procesos de aprendizaje. Cerramos este artículo con dos nuevas preguntas: ¿las analíticas al servicio de qué tipo de aprendizaje?, ¿pueden las analíticas contribuir hacia el aprendizaje significativo?

\section{Referencias bibliográficas}

Arnold, K.E. (2010). Signals: Applying academic analytics. Educause Quarterly, 33(1), 10.

Baker, R. S. J. D., \& Yacef, K. (2009). The state of educational data mining in 2009: A review and future visions. Journal of Educational Data Mining, 1(1), 3-17

Becerra, M. (2010). Mutaciones en la superficie y cambios estructurales. América Latina en el Parnaso informacional. Dénis De Moraes (comp.), Mutaciones de lo invisible. Comunicación y procesos culturales en la era digital, Buenos Aires, Paidós, 81-113.

Borgatti, S. P., Mehra, A., Brass, D. J., \& Labianca, G. (2009). Network analysis in the social sciences. Science, 323(5916), 892-895. 
Buckingham, S. (2012). Learning Analitics. Policy Brief, UNESCO Instituto para las Tecnologías de la Información en Educación. Recuperado de https://bit.ly/2TXK1rh.

Buckingham, S, \& Deakin, R. (2016). Learning analytics for 21 st century competencies. Journal of Learning Analytics, 3(2), 6-21. Recuperado de https://bit.ly/2W581FB.

Buckingham, S, \& Deakin, R. (2012). Learning dispositions and transferable competencies: pedagogy, modelling and learning analytics. In Proceedings of the 2nd international conference on learning analytics and knowledge (pp. 92-101). ACM. Recuperado de https://bit.ly/2ZssaaN.

Buckingham, S., \& Ferguson, R. (2012). Social Learning Analytics. Educational Technology \& Society, 15(3), 3-26. Recuperado de https://bit.ly/2UB/weH.

Burburles, N.C., y Callister, T. A. (2001). Educación: riesgos y promesas de las nuevas tecnologías de la información. Barcelona: Granica.

Clauset, A., Newman, M. E. J., \& Moore, C. (2004). Finding community structure in very large networks. Physical Review E, 70(6).

Coll, C., Engel, A., Niño, S. (2017). La actividad de los participantes como fuente de información para promover la colaboración. Una analítica del aprendizaje basada en el modelo de Influencia Educativa Distribuida. RED. Revista de Educación a Distancia, 53. DOI: http://dx.doi.org/10.6018/red/53/2 Recuperado de http://www.um.es/ead/ red/53/coll et al.pdf.

De-la-Fuente-Valentín, L., y Burgos, D. (2015). A4Aprendizaje: Un enfoque metodológico iterativo para apoyar mejor el aprendizaje y la enseñanza. IEEE Latin America Transactions, 13(2), 483-489.

De Laat, M., Lally, V., Lipponen, L., \& Simons, R.-J. (2006). Analysing student engagement with learning and tutoring activities in networked learning communities: A multi-method approach. International Journal of Web Based Communities, 2(4), 394-412.

Doueihi, M. (2010). La gran conversión digital. Buenos Aires: Fondo de Cultura Económica.

Durall, E., Gros, B., Maina, M., Johnson, L. \& Adams, S. (2012). Perspectivas tecnológicas: educación superior en Iberoamérica 2012-2017. Austin, Texas: The New Media Consortium. Recuperado de https://bit.ly/1jfsDrm

Fidalgo-Blanco, Á., Sein-Echaluce, M. L., García-Peñalvo, F. J., \& Conde, M. Á. (2015). Using Learning Analytics to improve teamwork assessment. Computers in Human Behavior, 47, 149-156.

Fortunato, S. (2010). Community detection in graphs. Physics Reports, 486(3-5), 75-174.

García-Holgado, A., \& García-Peñalvo, F. J. (2017). A metamodel proposal for developing learning ecosystems. In P. Zaphiris \& A. Ioannou (Eds.), Learning and Collaboration Technologies. Technology in Education. 4th International onference, LCT 2017. Held as Part of HCl International 2017, Vancouver, BC, Canada, July 9-14, 2017. Proceedings, Part I (pp. 100-109). Switzerland: Springer International Publishing. doi: 10.1007/978-3-319-58509-3_10

Gómez-Aguilar, D.A., García-Peñalvo, F.J., y Therón, R. (2014). Analítica visual en e-learning. El profesional de la información, 23(3). Recuperado de https://bit.ly/2IUu9yP.

Haythornthwaite, C., \& de Laat, M. (2010). Social networks and learning networks: Using social network perspectives to understand social learning. Paper presented at the 7th International Conference on Networked Learning, Aalborg, Denmark. 
Hirst, A. J. (2011). Social networks on delicious. https://bit.ly/2EmFpRr

Jones, C., \& Steeples, C. (2003). Perspectives and issues in networked learning. In C. Steeples \& C. Jones (Eds.), Networked Learning: Perspectives and Issues. Lancaster: Centre for Studies in Advanced Learning Technology

Kruse, A., y Pongsajapan, R. (2016). Student-Centered Learning Analytics. CNaLS. Thought Papers. Recuperado de https://bit.ly/2GEmFi3.

Levine, A. (2006) Educación Superior: Una Revolución Interna y Otra Externa. In Serbin Pittinsky, Matthew. La Universidad conectada (pp.25-42). Málaga: El Aljibe.

Lévy, P. (2007). Cibercultura. La cultura de la sociedad digital. Barcelona: Anthropos.

Long, P, \& Siemens, G. (2011). Penetrating the Fog: Analytics in Learning and Education, Educause Review, 46(5), 31-40. Recuperado de https://bit.ly/2UvNXuA.

Mazza, R., \& Dimitrova, V. (2007). CourseVis: A graphical student monitoring tool for supporting instructors in web-based distance courses. International Journal of Human-Computer Studies, 65(2), 125-139.

Mitchell, J., \& Costello, S. (2000). International e-VET market research report: A report on international market research for Australian VET online products and services. Sydney, Australia: John Mitchell \& Associates and Education Image.

Miege, B. (2010) La cuestión de las TIC: hacia nuevos planteamientos. In D. de Moraes (org.) Mutaciones de lo visible. Comunicación y procesos culturales en la era digital, (pp. 15-44). Bs.As: Paidós.

Piatetsky-Shapiro, G. (1995). Guest editor's introduction: Knowledge discovery in databases from research to applications. Journal of Intelligent Information Systems, 4(1), 5-6

Pistilli, M., \& Arnold, K. (2012). Course signals at Purdue: Using learning analytics to increase student success. Paper presented at the 2 nd International Conference on Learning Analytics and Knowledge, Vancouver, Canada.

Reffay, C., \& Chanier, T. (2003). How social network analysis can help to measure cohesion in collaborative distance-learning. International Conference on Computer Supported Collaborative Learning (pp. 243-352). Bergen: Kluwer Academic Publishers.

Rodríguez-Triana, M.J., Martínez-Monés, A., Asensio-Pérez, J.I., y Dimitriadis, Y. (2015). Las secuencias de comandos y la supervisión se encuentran entre sí: alineando el análisis de aprendizaje y el diseño de aprendizaje para apoyar a los maestros en la organización de situaciones CSCL. Revista británica de tecnología educativa, 46 (2), 330-343.

Romero, C., \& Ventura, S. (2007). Educational data mining: A survey from 1995 to 2005. Expert Systems with Applications, 33(1), 135-146.

Sadin, E. (2013). Las tecnologías digitales debilitan la capacidad de decidir. Diario Página 12. Recuperado de https://bit.ly/2PzQROh.

Siemens, G. (2013). Learning Analytics: The Emergence of a Discipline, American Behavioral Scientist, 57(10), 1380-1400, SAGE Publications. Recuperado de https://bit. Iy/2Puq7P8.

Siemens, G., Gasevic, D., Haythornthwaite, C., Dawson, S., Buckingham, S., Ferguson, R., Duval, E., Verbert, K., Baker, R, S.J.d . (2011). Open learning analytics: an integrated \& modularized platform. Proposal to design, implement and evaluate an open platform 
to integrate heterogeneous learning analytics techniques. Recuperado de https:// bit.ly/2PoQwOH.

Siemens, G., Baker, R. S.J.d. (2012). "Learning analytics and educational data mining: towards communication and collaboration". En: Proceedings of the 2nd International Conference on Learning Analytics and Knowledge (Vancouver, Canada), pp. 252-254.

Suthers, D., \& Verbert, K. (2013, April). Learning analytics as a middle space. In Proceedings of the Third International Conference on Learning Analytics and Knowledge (pp. 1-4). ACM. Recuperado de https://bit.ly/2UCEYHY.

Tobeña, V. (2011). La escuela en el mundo contemporáneo. Notas sobre el cambio cultural. In G. Tiramonti (org.). Variaciones sobre la forma escolar. Límites y posibilidades de la escuela media. Bs.As.: Homosapiens.

UNESCO (1998). Conferencia Mundial sobre la Educación Superior. La educación superior en el siglo XXI: Visión y acción. 9 de octubre de 1998. Recuperado de https://bit. ly/1blr26L.

Wasserman, S., \& Faust, K. (1994). Social network analysis: Methods and applications (Structural analysis in the social sciences). Cambridge: Cambridge University Press 